\title{
Update on the management of invasive bladder cancer 2012
}

This article was published in the following Dove Press journal:

Cancer Management and Research

17 July 2012

Number of times this article has been viewed

\author{
Hans Goethuys' \\ Hein Van Poppel ${ }^{2}$ \\ 'Department of Urology, Ziekenhuis \\ Oost-Limburg, Genk, Belgium; \\ ${ }^{2}$ Department of Urology, University \\ Hospital Leuven, Leuven, Belgium
}

\begin{abstract}
Muscle-invasive bladder cancer is a deadly disease for which a number of new approaches have become available to improve prognosis. A recent review emphasized the importance of timely indication of surgery and highlighted current views regarding the adequate extent of the surgery and the importance of lymph node dissection. Furthermore, treatment using neoadjuvant and adjuvant systemic chemotherapy has become more prominent, while cystectomy and diversion should be conducted only in experienced centers. Optimal methods of urinary diversion and the use of robot-assisted laparoscopic cystectomy require further study.

Keywords: bladder cancer, surgery, chemotherapy, urinary diversion
\end{abstract}

\section{Introduction}

Multiple treatment options are available for managing muscle-invasive bladder cancer. However, radical cystectomy is considered the gold standard for treating this disease. The aim of this invasive surgery is to remove all cancer in the bladder, pelvis, and regional lymph nodes. On average, radical cystectomy is unsuccessful in approximately $50 \%$ of patients; it is therefore essential that efforts are made to improve the outcomes of this surgery, by way of determining a timely indication for surgery, defining an adequate extent of surgery, and proposing combinations with systemic chemotherapy. Furthermore, invasive bladder cancer surgery should be conducted in experienced and high-volume centers.

\section{Timely indication for cystectomy}

The prognosis for muscle-invasive bladder cancer in patients with an early-stage disease and negative lymph nodes is good; thus, early cystectomy for low-stage tumors will give excellent results. However, non-muscle-invasive bladder cancer is not subjected to radical cystectomy but to the more conservative transurethral resection and intravesical chemotherapy or immunotherapy. Following the European Association of Urology (EAU) guidelines, T1 tumors invading the stroma and those at high risk of progression (eg, high grade, multifocality, carcinoma in situ, and large tumor size), and all T1 tumors failing intravesical therapy are considered for early cystectomy. ${ }^{1}$ Additionally, some patients with extensive papillary tumors that cannot be controlled through transurethral resection and intravesical therapy alone can become candidates for cystectomy. However, once the tumor invades the muscularis propria of the detrusor muscle, radical cystectomy is indicated. Early cystectomy should be promoted not only to improve oncologic outcomes but also to increase the potential 
for functional restoration through orthotopic substitution and nerve sparing.

Indeed, large patient series have demonstrated that disease-free survival following cystectomy is between 48 and $68 \% .^{2,3}$ Outcomes improve when tumors are confined to an organ compared to tumors invading extravesically or invading lymph nodes.

Another factor in the timely performance of radical cystectomy emerges from several studies revealing that in patients with muscle-invasive cancer at diagnosis, delaying surgery for 90 days is associated with a significant decrease in survival probability. ${ }^{4-6} \mathrm{~A}$ delay in cystectomy after diagnosis of muscle-invasive bladder cancer may, however, be justified when neoadjuvant therapy is delivered, although this carries an oncologic risk. Timely indication and performance of surgery for muscle-invasive bladder cancer is clearly an important prognostic factor.

\section{Adequate extent of surgery}

In addition to radical cystectomy (with or without systemic chemotherapy), another curative treatment is a bladdersparing approach consisting of a thorough transurethral bladder tumor resection, radiation treatment, and systemic chemotherapy. ${ }^{7}$ No prospective randomized studies to compare these two strategies are available, but a Cochrane database analysis of individual trials suggested a benefit of radical surgery over radiation. ${ }^{8}$ Thus, in patients that are able to undergo surgery, this remains the treatment of choice.

In male patients, radical cystectomy refers to radical cystoprostatectomy with or without resection of the urethra. Resection of the urethra is mandatory when there is an obvious invasion of the urethra or of the prostate. Many years ago, several risk factors for urethral recurrence after cystectomy without urethrectomy were recognized, including carcinoma in situ, multifocal transitional cell carcinoma (TCC), TCC at the bladder neck or in the upper tract, and TCC in the prostatic urethra. More recent work, however, has shown that invasion of the mucosa or even the ducts of the prostatic urethra are not a contraindication for urethral preservation, and that only invasion of the prostatic stroma by TCC is an absolute contraindication for bladder replacement. ${ }^{9}$ Thus, in cases of urethral involvement or invasion in the prostatic stroma, prophylactic urethrectomy must be performed. The overall recurrence rate at the level of the urethra left in place is estimated to be between $4 \%$ and $10 \%$. When urethral recurrence is detected, the patient is typically already in a metastastic stage. When a urethra remains, follow-up with cystoscopy and cytology is required. Delayed urethrectomy for tumor recurrence is difficult. Thus, the urethra should be removed when it is defunctionalized, and patients undergoing cutaneous diversion should not receive bladder replacement. Therefore, many experts advocate for simultaneous urethrectomy in all patients undergoing cutaneous diversion. ${ }^{10}$ In patients that are candidates for orthotopic bladder replacement, the prostatic urethra can be sampled before surgery is planned or a frozen section can be taken of the urethral section margin during the surgery to determine whether ureterectomy is mandatory. In female patients, radical cystectomy involves anterior exenteration with resection of the internal genitalia (hysterectomy and ovariectomy), resection of the anterior vaginal wall, and cystourethrectomy. Additionally, when bladder neck or urethra is free of the tumor, the urethra can be preserved and bladder replacement can be proposed. ${ }^{11}$

Nerve-sparing cystoprostatectomy has become increasingly popular since it allows patients to recover erectile function 8 to 9 months after surgery. Some physicians have advocated prostate sparing cystectomies, ${ }^{12}$ while others feel that this procedure is completely inappropriate in patients undergoing cystectomy for transitional cell carcinoma. ${ }^{13}$ Proper nerve-sparing cystectomy techniques eliminate the need for this oncologically unsafe procedure. ${ }^{8}$

During cystectomy, most urologists perform a frozen section of the margin of the ureters, although there is no conclusive evidence regarding the usefulness of this method. In cases of obvious TCC or carcinoma in situ on frozen section analysis, higher ureterectomy can be performed. A recent study reported a high sensitivity and specificity of frozen section analysis to reduce the risk of positive ureteral margins, while positive final ureteral margins were significantly associated with subsequent upper tract recurrence. ${ }^{14}$ In all cases of simultaneous upper tract TCC, very careful follow-up is mandatory.

Controversy exists regarding the required extent of lymphadenectomy and the number of nodes that must be removed during classical radical cystectomy. Some groups conduct intrapelvic lymph node dissection limited to the obturator fossa, others to the obturator and internal iliac or external iliac area, whereas others conduct more extended lymph node dissections, including the common iliac to the aortic bifurcation. The extent of dissection as well as the number of harvested nodes varies widely, from 2 or 3 up to 30 nodes per specimen. Not only the number of nodes analyzed, but also the method pathologists use to conduct detailed analysis of the specimen appears to be important; therefore, surgeons are advised to deliver lymphadenectomy specimens in different 
marked flasks to identify where an eventual positive node was located..$^{15}$

Lymph node dissection has an obvious diagnostic role, and a higher number of nodes removed will result in more correct staging. Lymph node dissection has also been claimed to have a therapeutic role. In a direct sense, lymph node dissection can be used to remove microscopic, node-confined, metastatic disease. ${ }^{15}$ In contrast, an indirect therapeutic role can be observed since some degree of nodal invasion can indicate the application of adjuvant chemotherapy.

Lymph node density is defined as the number of positive nodes relative to the number of nodes removed. At least two studies have demonstrated survival improvement with an increased number of nodes removed and survival decrease with a higher number of positive nodes. ${ }^{16,17}$ Both studies showed a statistically significant difference depending on whether more or less than 20 percent of nodes were invaded.

An interesting comparative study between two highvolume centers, the Cleveland Clinic in USA and the Inselspital in Berne, provided insight into the therapeutic benefit of pelvic lymph node dissection. In the Cleveland Clinic, patients had limited lymph node dissection, while those in Berne underwent extended lymph node dissection. From this comparative study, it was clear that in the Cleveland Clinic, patients fared statistically significantly worse than those in the Berne cohort, demonstrating a significant survival benefit of extended pelvic lymph node dissection over limited dissection. ${ }^{18}$ Another single-center study from Mansoura, Egypt, showed that the extent of lymphadenectomy in radical cystectomy for bladder cancer influences disease-free survival. ${ }^{19}$ This study showed that extended lymphadenectomy is associated with better disease-free survival for bladder cancer patients with "endopelvic" lymph node involvement and therefore extended lymphadenectomy should be considered in this category of patients.

The exact template for extended pelvic lymph node dissection has not yet been clearly defined, but based upon reports from high-volume centers, the template should include external and internal iliac vessels, obturator fossa, and common iliac vessels up to the ureter crossing. Some authors suggest that this dissection should be extended cranially up to the aortic bifurcation in lymph node positive cases, but the oncologic benefit of this method has not been shown.

\section{Combination with systemic chemotherapy}

Radical cystectomy is quite an invasive procedure and exhibits significant complications; this method remains unsatisfactory and little progress has been made to correct these issues in recent years. Combination with systemic chemotherapy, applied after surgery in patients with poor prognostic factors and later before surgery in high-risk patients, has been explored. Most studies have involved a small number of patients and have used different chemotherapy regimens, including cisplatin monotherapy. The Advanced Bladder Cancer Meta-analysis collaboration, which was published in 2005, provided the results of all valuable studies examining neo-adjuvant and adjuvant chemotherapy with survival as an endpoint. Neo-adjuvant chemotherapy using cisplatin monotherapy did not increase survival, but platinum-based polychemotherapy resulted in significantly improved survival when seven neoadjuvant study results were pooled. ${ }^{20}$ Fewer studies are available examining adjuvant chemotherapy. Cisplatin monotherapy yielded no benefit, but in the five platinumbased polychemotherapy studies, adjuvant chemotherapy improved survival. ${ }^{14}$ Differences were statistically significant for both approaches. ${ }^{21}$ Despite these results, neo-adjuvant chemotherapy has not increased in popularity in Europe, and in many centers, adjuvant chemotherapy is preferred in young patients, those with locally advanced and high-grade tumors, and those with or without nodal involvement. $^{22}$

Recently, the Journal of Clinical Oncology published the results of a neo-adjuvant triple chemotherapy combination involving cisplatin, methotrexate, and vinblastine (CMV). This intergroup study (EORTC 30894/MRC BA06) was reanalyzed for long-term efficacy of these treatments. The final patients were included in 1995 and a median followup of 8 years has been reached. Patients had T2G3, T3, or T4a tumors with or without nodal invasion, but clinically, patients reached $\mathrm{M} 0$ before curative cystectomy or full-dose external beam radiation therapy. Thus, both local treatments were included in the study. A total of 491 patients received three cycles of neoadjuvant CMV, while 485 patients received no chemotherapy. The risk of death decreased by $16 \%$ in the neoadjuvant chemotherapy group $(P=0.037)$. Ten-year survival improved following CMV by a statistically significant value of $6 \%(P<0.05)$. The same advantage with neoadjuvant chemotherapy was observed, not only for overall survival but also for metastasis-free survival, local recurrence disease-free survival, and disease-free survival. When overall survival and local recurrence disease-free survival were compared between patients treated with cystectomy or radiation, cystectomy was favored for both endpoints. $^{23}$ 
These results should normally result in a paradigm shift in which all patients with muscle-invasive bladder cancer may become candidates for neoadjuvant chemotherapy. However, this does not occur in practice and many urological surgeons continue advocating cystectomy alone in patients with smaller muscle-invasive bladder cancers, particularly those that are not deeply invasive. However, a defined subgroup of bladder tumors with prognosis has not been established. Classically, methotrexate, vinblastine, doxorubicin, and cisplatin (MVAC) therapy has been used in the neoadjuvant setting before cystectomy. More recently, gemcitabine + cisplatin (GC) have been used because this combination is associated with less non-hematologic toxicity than MVAC with a comparable downstaging effect. ${ }^{24}$ Despite the lack of data regarding the relative efficacy of GC versus MVAC in the neoadjuvant setting, these regimens have been used interchangeably. A recent investigation did not observe cisplatin-based regimen to be an independent predictor of survival. A trend was observed towards improved survival and a greater complete response rate in the MVAC group. ${ }^{25}$

The EAU Guidelines 2010 state that "neo-adjuvant Cisplatin-containing combination chemotherapy should be considered in muscle invasive bladder cancer, irrespective of the definitive treatment." 10 In contrast, it remains clear that neoadjuvant chemotherapy is not recommended in older patients with poor performance status or in patients with impaired renal function.

\section{Cystectomy in experienced centers}

A final but important factor that can contribute to optimal oncological results may be the concentration of cystectomy procedures in centers with experienced surgeons and high volumes. Barbieri et $\mathrm{al}^{26}$ showed that mortality following cystectomy is strongly related to the number of cystectomies performed by the center per year. The authors analyzed more than 6700 cystectomies performed between 2002 and 2005 . In centers performing less than 10 cystectomies per year, postoperative mortality was twice that in centers performing more than 25 cystectomies per year. In those performing more than 50 per year, mortality was less than $0.5 \%$. Other authors have investigated this procedure in 133 hospitals in Texas, where mortality was analyzed after 1302 cystectomies. ${ }^{27} \mathrm{In}$ low- and moderate-volume centers (less than 10 cystectomies per year), mortality was approximately $3 \%$, while in centers performing more than 10 cystectomies per year mortality was $0.7 \%$. This is clearly a factor that patients should consider, and insurance companies should conduct in-depth analysis of these figures. Therefore, urologists should be aware of their own figures and critically analyze whether a sufficient number of procedures are performed to maintain their surgical skills and lower complication rates.

\section{What type of urinary diversion?}

The ideal urinary diversion after radical cystectomy should be easy, exhibit minimal morbidity and mortality, should be protective for the upper tract, and be well-perceived by the patient. Continent diversions to the skin (Indiana pouch, Mainz pouch, Monti stomas) clearly have an advantage in that no bag must be applied to the abdominal wall, but it remains questionable whether this guarantees a better quality of life. The disadvantages of this method include the need for self-catheterization, the possibility of leakage (which is difficult to cope with in cases of umbilical diversion), difficulties with catheterization, stenosis and kinking, and the requirement for reoperations in approximately $30 \%$ of cases. Bladder substitution procedures, when they are oncologically safe, have the advantage of allowing patients to continue voiding through the urethra, which may have some impact on the quality of life. Disadvantages include the possibility of urinary stress incontinence; however, and even more embarrassing, hypercontinence can occur, which is much more frequent in females than in males but can occur in both genders. Additionally, enuresis nocturna is a well-documented and frequently occurring consequence of bladder replacement. During the immediate postoperative period, patients must be aware of potential problems such as metabolic acidosis and diarrhea that may require medical attention.

The Cochrane collaboration compared ileal conduits to orthotopic bladder and continent diversion to the skin and evaluated early complications, upper tract preservation, urinary tract infections, and quality of life. ${ }^{28}$ The analysis included 18 trials, two randomized clinical trials addressing selected criteria, one randomized clinical trial with no numbers, two randomized clinical trials addressing unselected criteria, and 13 nonrandomized trials. Unfortunately, the superiority of one treatment over the others could not be shown. Therefore, no specific recommendation exists for diversion type. Diversion type should be decided preoperatively and should take into account the age and performance status of the patient, T stage, and renal function (eGFR). Young patients concerned with body image can, after sufficient information regarding complication rates, be offered a continent cutaneous diversion preferably at the level of the umbilicus because of cosmetic reasons. Patients 
up to 70 years of age for which the urethra can be preserved can be offered bladder substitution; this group of patients with bladder cancer is continually increasing. However, older patients with advanced stage disease and shorter life expectancy or compromised renal function will continue to be offered an ileal conduit, which remains the safest and most well-accepted form of urinary diversion. ${ }^{29}$

\section{Robot-assisted laparoscopic cystectomy?}

An increasing amount of data regarding the outcome of minimal invasive surgical techniques have become available for invasive bladder cancer. Open cystectomy has the advantage of being a procedure with a proven oncological efficacy, the possibility of tactile feedback, and an easily performed extended lymph node dissection. Open cystectomy, in contrast, is associated with increased bleeding and slower overall physical recovery. Laparoscopic (with or without robot) cystectomy can be performed with less bleeding, less postoperative pain, and earlier recovery, but the long-term oncological outcomes remain uncertain, and the duration and cost of the procedure remains an obstacle. ${ }^{30}$ Nevertheless, robotic surgery, not only for bladder cancer but also for other diseases, continues to evolve. A comprehensive electronic literature search showed encouraging short- to medium-term results in terms of postoperative morbidity and oncologic outcomes. It seems possible in experienced hands to satisfactorily perform minimally invasive lymphadenectomy. Positive soft tissue margins are similar to large open series for T2-T3 disease, but inferior for bulky $\mathrm{T} 4$ disease. The concerns regarding the ability of minimal invasive radical cystectomy to treat bulky and locally advanced disease makes careful patient selection a critical factor. ${ }^{31}$

\section{Conclusion}

From the present literature the following statements can be derived:

1. Radical cystectomy involving extended pelvic lymph node dissection is the treatment of choice for muscleinvasive bladder cancer.

2. Curing bladder cancer is possible, but requires timely indication and nondelayed performance of cystectomy.

3. When the urethra is not used for bladder replacement, it should be removed.

4. Patients with deep muscle-invasive bladder cancer should be counseled for neoadjuvant cisplatin-based chemotherapy.
5. Radical cystectomy should be conducted in high-volume centers.

6. Different urinary diversion types show good results in properly selected patients, and a Bricker diversion is often an easy, safe, and acceptable solution.

7. Robotic surgery will continue to evolve, but studies to demonstrate its oncological equivalence to traditional techniques should be performed.

\section{Disclosure}

The authors report no conflicts of interest in this work.

\section{References}

1. Babjuk M, Oosterlinck W, Sylvester R, et al; European Association of Urology (EAU). EAU guidelines on non-muscle-invasive urothelial carcinoma of the bladder, the 2011 update. Eur Urol. 2011;59:997-1008.

2. Stein JP, Lieskovsky G, Cote R, et al. Radical cystectomy in the treatment of bladder cancer: long-term results in 1,054 patients. $J$ Clin Oncol. 2001;19:666-675.

3. Ghoneim MA, Abdel-Latif M, el-Mekresh M, et al. Radical cystectomy for carcinoma of the bladder: 2,720 consecutive cases 5 years later. J Urol. 2008;180:121-127.

4. Sánchez-Ortiz RF, Huang WC, Mick R, Van Arsdalen KN, Wein AJ, Malkowicz SB. An interval longer than 12 weeks between the diagnosis of muscle invasion and cystectomy is associated with worse outcome in bladder carcinoma. $J$ Urol. 2003;169:110-115.

5. Mohmud SM, Fong B, Fahmy N, Tanquay S, Aprikian AG. Effect of preoperative delay on survival in patients with bladder cancer undergoing cystectomy in Quebec: a population based study. $J$ Urol. 2006;175:78-83.

6. Lee CT, Madii R, Daignault S, et al. Cystectomy delay more than 3 months from initial bladder cancer diagnosis results in decreased disease specific and overall survival. $J$ Urol. 2006;175:1262-1267.

7. Efstathiou JA, Spiegel DY, Shipley WU, et al. Long-term outcomes of selective bladder preservation by combined-modality therapy for invasive bladder cancer: the MGH experience. Eur Urol. 2012;61: 705-711.

8. Shelley MD, Wilt TJ, Barber J, Mason MD. A meta-analysis of randomised trials suggests a survival benefit for combined radiotherapy and radical cystectomy compared with radical radiotherapy for invasive bladder cancer: are these data relevant to modern practice? Clin Oncol (R Coll Radiol). 2004;16:166-171.

9. Hardeman SW, Soloway MS. Urethral recurrence following radical cystectomy. J Urol. 1990;144:666-669.

10. Van Poppel H, Joniau S, Groen LA. Prepubic urethrectomy. BJU Int 2008;103:118-132.

11. Stenzl A, Cowan NC, De Santis M, et al. Treatment of muscle-invasive and metastatic bladder cancer: update of the EAU guidelines. Eur Urol. 2011;59:1009-1018.

12. Nieuwenhuijzen JA, Meinhardt W, Horenblas S. Clinical outcomes after sexuality preserving cystectomy and neobladder (prostate sparing cystectomy) in 44 patients. J Urol. 2005;173;1314-1317.

13. Hautmann RE, Stein JP. Neobladder with prostatic capsule and seminalsparing cystectomy for bladder cancer; a step in the wrong direction. Urol Clin North Am. 2005;32:177-185.

14. Gakis G, Schilling D, Perner S, Schwentner C, Sievert KD, Stenzl A. Sequential resection of malignant ureteral margins at radical cystectomy: a critical assessment of the value of frozen section analysis. World J Urol. 2011;29:451-456.

15. Karl A, Carroll PR, Geschwend JE, et al. The impact of lymphadenectomy and lymph node metastasis on the outcomes of radical cystectomy for bladder cancer. Eur Urol. 2009;55:826-835. 
16. Stein JP, Cai J, Groshen S, Skinner DG. Risk factors for patients with pelvic node metastases following radical cystectomy with en block pelvic lymphadenectomy: the concept of lymph node density. J Urol. 2003;170:35-41.

17. Fleischmann A, Thalmann GN, Markwalder R, Studer UE. Extracapsular extension of pelvic lymph node metastases from urothelial carcinoma of the bladder in an independent prognostic factor. $J$ Clin Oncol. 2005;23:2358-2365.

18. Dhar NB, Klein EA, Reuther AM, Thalmann GN, Madersbacher S, Studer UE. Outcome after radical cystectomy with limited and extended pelvic lymph node dissection? J Urol. 2008;179:873-878.

19. Abol-Enein H, Tilki D, Mosbah A, et al. Does the extent of lymphadenectomy in radical cystectomy for bladder cancer influence diseasefree survival? A prospective single-center study. Eur Urol. 2011;60: $572-577$.

20. Advanced Bladder Cancer Meta-analysis Collaboration. Neoadjuvent chemotherapy in invasive bladder cancer: a systematic review and meta-analysis. Lancet. 2003;361:1927-1934.

21. Advanced Bladder Cancer (ABC) Meta-analysis Collaboration. Adjuvant chemotherapy in invasive bladder cancer: a systematic review and meta-analysis of individual patient data Advanced Bladder Cancer (ABC) Meta-analysis Collaboration. Eur Urol. 2005;48:189-199.

22. Sonpavde G, Petrylak DP. Perioperative chemotherapy for bladder cancer. Crit Rev Oncol Hematol. 2006;57:133-144.

23. International Collaboration of Trialists, Medical Research Council Advanced Bladder Cancer Working Party (now the National Cancer Research Institute Bladder Cancer Clinical Studies Group, European Organisation for Research and Treatment of Cancer Genito-Urinary Tract Cancer Group, et al. International phase III trial assessing neoadjuvant cisplatin, methotrexate, and vinblastine chemotherapy for muscle-invasive bladder cancer: long-term results of the BA06 30894 trial. J Clin Oncol. 2011;29:2171-2177.
24. Kaneko G, Kikuchi E, Matsumoto K, Obata J, et al. Neoadjuvant gemcitabine plus cisplatin for muscle-invasive bladder cancer. Jpn J Clin Oncol. 2011;41:908-914.

25. Yeshchina O, Badalato GM, Wosnitzer MS, et al. Relative efficacy of perioperative gemcitabine and cisplatin versus methotrexate, vinblastine, adramycin, and cisplatin in the management of locally advanced urothelial carcinoma of the bladder. Urology. 2012;79:384-390.

26. Barbieri CE, Lee B, Cookson MS, et al. Association of procedure volume with radical cystectomy outcomes in a nationwide database. J Urol. 2007;178:1418-1421.

27. Elting LS, Pettaway C, Bekele BN, et al. Correlation between annual volume of cystectomy, professional staffing, and outcomes: a statewide, population-based study. Cancer. 2005;104(5):975-984.

28. Nabi G, Yong SM, Ong E, McPherson G, Grant A, N'Dow J. Is orthotopic bladder replacement the new gold standard? Evidence from a systematic review. J Urol. 2005;174:21-28.

29. World Health Organization (WHO) Consensus Conference on Bladder Cancer, Hautmann RE, Abol-Enein H, et al. Urinary diversion. Urology. 2007;69(Suppl 1):17-49.

30. Khan MS, Challacombe B, Elhage O, et al. A dual-centre, cohort comparison of open laparoscopic and robotic-assisted radical cystectomy. Int J Clin Pract. April 16, 2012 [Epub ahead of print.]

31. Challacombe BJ, Bochner BH, Dasgupta P, et al. The role of laparoscopic and robotic cystectomy in the management of muscleinvasive bladder cancer with special emphasis on cancer control and complications. Eur Urol. 2011;60:767-775.
Cancer Management and Research

\section{Publish your work in this journal}

Cancer Management and Research is an international, peer-reviewed open access journal focusing on cancer research and the optimal use of preventative and integrated treatment interventions to achieve improved outcomes, enhanced survival and quality of life for the cancer patient. The journal welcomes original research, clinical \& epidemiological

\section{Dovepress}

studies, reviews \& evaluations, guidelines, expert opinion \& commentary, case reports \& extended reports. The manuscript management system is completely online and includes a very quick and fair peerreview system, which is all easy to use. Visit http://www.dovepress.com/ testimonials.php to read real quotes from published authors. 